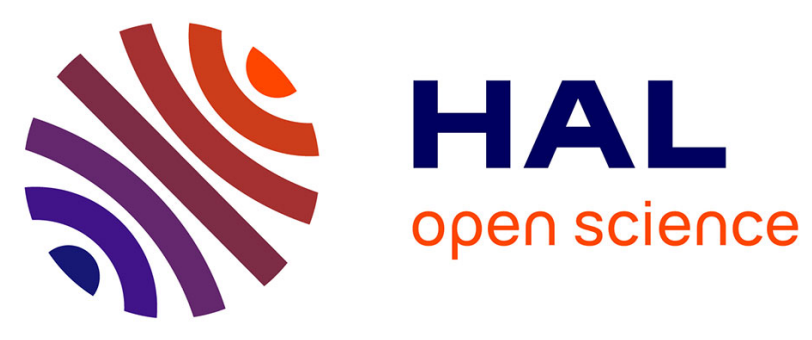

\title{
Multidimensional Low-Rank Filter based on the AU-HOSVD for MIMO STAP
}

Maxime Boizard, Frédéric Brigui, Guillaume Ginolhac, Frédéric Pascal, Philippe Forster, Hong Bo Sun

\section{- To cite this version:}

Maxime Boizard, Frédéric Brigui, Guillaume Ginolhac, Frédéric Pascal, Philippe Forster, et al.. Multidimensional Low-Rank Filter based on the AU-HOSVD for MIMO STAP. 2013 IEEE 5th International Workshop on Computational Advances in Multi-Sensor Adaptive Processing (CAMSAP 2013), Dec 2013, Saint Martin, France. pp.1-4, 10.1109/CAMSAP.2013.6714095 . hal-00933556

\section{HAL Id: hal-00933556 https://hal.science/hal-00933556}

Submitted on 20 Jan 2014

HAL is a multi-disciplinary open access archive for the deposit and dissemination of scientific research documents, whether they are published or not. The documents may come from teaching and research institutions in France or abroad, or from public or private research centers.
L'archive ouverte pluridisciplinaire HAL, est destinée au dépôt et à la diffusion de documents scientifiques de niveau recherche, publiés ou non, émanant des établissements d'enseignement et de recherche français ou étrangers, des laboratoires publics ou privés. 


\title{
Multidimensional Low-Rank Filter based on the AU-HOSVD for MIMO STAP
}

\author{
Maxime Boizard ${ }^{* \S}$, Frédéric Brigui ${ }^{\dagger}$, Guillaume Ginolhac ${ }^{\ddagger}$, Frédéric Pascal ${ }^{\S}$, Philippe Forster* and Hong Bo Sun ${ }^{\dagger}$ \\ *Satie Laboratory, ENS Cachan - CNRS - UniverSud, Cachan 94235, France \\ Email: maxime.boizard,philippe.forster@satie.ens-cachan.fr \\ †Temasek Laboratories@NTU, Nanyang Technological University Singapore 637553. \\ Email: frederic.brigui,EHBSun@ntu.edu.sg \\ ${ }^{\ddagger}$ LISTIC, Polytech Annecy-Chambéry, Annecy-le-Vieux, France. Email: guillaume.ginolhac@univ-savoie.fr \\ §SONDRA Laboratory, Supélec, Gif-sur-Yvette, France. Email: frederic.pascal@supelec.fr
}

\begin{abstract}
We propose in this paper a new low rank filter for MIMO STAP (Multiple Input Multiple Output Space Time Adaptive Processing) based on the AU-HOSVD (Alternative Unfolding Higher Order Singular Value Decomposition). This decomposition called the AU-HOSVD is able to process data in correlated dimensions which is desirable for STAP methods. We apply the new filter to MIMO STAP simulated data. The results are encouraging and outperforms the conventional STAP 2D filter in terms of number of secondary data.
\end{abstract}

\section{INTRODUCTION}

In signal processing, more and more applications deal with multidimensional data whereas most of the signal processing algorithms are based on one or two dimensional models. Consequently, multidimensional data have to be folded as vector or matrix to be processed. The multidimensional structure of the data is then not exploited. The multilinear algebra [1], [2] provides a good framework to exploit these data by preserving the structure information. In this context, data are represented as multidimensional arrays called tensor. However, generalizing matrix-based algorithms to the multilinear algebra framework is not a trivial task.

Let us consider the case of the Singular Value Decomposition (SVD). There is no single multilinear extension of the SVD, with exactly the same properties as the SVD. Depending on which properties are preserved, several extensions of the SVD have been introduced as for example the Higher Order Singular Value Decomposition (HOSVD) [2]. The HOSVD is based on the classic tensor unfolding. This unfolding transforms a tensor into a matrix in order to highlight one dimension. In other words, HOSVD only considers simple information, which is the information contained in each dimension taken separately. The correlated information, which is the information contained in a combination of dimensions, is neglected. In order to use correlated information, we have previously proposed in [3], [4] a new set of orthogonal decompositions, the Alternative Unfolding HOSVD (AU-HOSVD). This new decomposition is then well adapted to applications for which the use of combined dimension is needed. This is the case for detection of moving target embedded in clutter using Space-Time Adaptive Processing (STAP) [5]. Indeed, both spatial and Doppler dimensions are needed to efficiently suppress the clutter. A new Low-Rank (LR) filter based on the HOSVD has been proposed in [3], [4] for polarimetric STAP data. This new filter shows good results, especially in terms of number of secondary data needed for the suppression of the clutter.

We propose in this paper to derived a LR filter based on the AU-HOSVD for MIMO STAP. MIMO radar [6] is a system with multiple transmit antennas and multiple receive antennas which transmit and receive multiple pulses for the detection of moving targets. Compared to conventional array, MIMO radar offers additional degrees of freedom and specifically waveform diversity for coherent MIMO where transmit and receive antennas are colocated (we only consider this configuration in this study). Specifically orthogonal waveforms allows to generate a long virtual antenna array and thus improve angular resolution. STAP processing has been naturally extended to MIMO radar [6]. Assuming ideally orthogonal waveforms, MIMO STAP allows narrower clutter rejection with the same number of antennas compared to the phased array. By nature, MIMO data are multidimensional. It seems that the LR filter based on the AU-HOSVD is well adapted to the MIMO STAP. The performances of the LR filter for the MIMO data are evaluated on simulated data.

The paper is organized as follows. Section II gives a brief overview of the HOSVD and the AU-HOSVD. In Section III we present the MIMO STAP and the derivation of the LR filter based on the AU-HOSVD and dedicated to MIMO data. Finally we apply and evaluate the proposed approach with simulated data.

The following convention is adopted: scalars are denoted as italic letters, vectors as lower-case bold-face letters, matrices as bold-face capitals, and tensors are written as bold-face calligraphic letters. We use the superscripts ${ }^{\dagger}$ for Hermitian transposition and ${ }^{*}$ for complex conjugation.

\section{TENSOR DECOMPOSITION}

In this section, we present an brief overview of the HOSVD and the new decomposition, the AU-HOSVD. More details on the AU-HOSVD and its application to polarimetric STAP can be found in [3], [4].

\section{A. Higher Order Singular Value Decomposition}

The Higher Order Singular Value Decomposition (HOSVD) is a particular case of Tucker decomposition [2] with orthogonality properties. We consider a tensor $\mathcal{H} \in \mathbb{C}^{I_{1} \times \ldots \times I_{P}}$ of order $P$. The HOSVD decomposes $\mathcal{H}$ as follows [2]:

$$
\mathcal{H}=\mathcal{K} \times_{1} \mathbf{U}^{(1)} \ldots \times_{P} \mathbf{U}^{(P)},
$$


where $\forall n, \mathbf{U}^{(n)} \in \mathbb{C}^{I_{n} \times I_{n}}$ is an orthonormal matrix and $\mathcal{K} \in \mathbb{C}^{I_{1} \times \ldots \times I_{P}}$ is the core tensor, which satisfies the all-orthogonality conditions [2]. The matrix $\mathbf{U}^{(n)}$ is given by the SVD of the $n$-dimension unfolding tensor, $[\mathcal{H}]_{n}=$ $\mathbf{U}^{(n)} \boldsymbol{\Sigma}^{(n)} \mathbf{V}^{(n) H}$. Using classical unfolding, the HOSVD only considers the information in each dimension separately. This may not be suitable for some applications that are based on the use of combined dimensions such as STAP.

\section{B. Alternative Unfolding HOSVD}

In order to use information of combined dimensions, we have proposed in [3], [4] a new decomposition called the AUHOSVD. We consider the same tensor $\mathcal{H}$ as previously. The entire set of dimensions of $\mathcal{H}$ is denoted by $\mathbb{A}=\{1, \ldots, P\}$ and a subset $l$ of $\mathbb{A}$ is denoted by $\mathbb{A}_{l}$. Then, the AU-HOSVD decomposes $\mathcal{H}$ as follows:

$$
\mathcal{H}=\mathcal{K}_{\mathbb{A}_{1} / \ldots / \mathbb{A}_{L}} \times_{\mathbb{A}_{1}} \mathbf{U}^{\left(\mathbb{A}_{1}\right)} \ldots \times_{\mathbb{A}_{L}} \mathbf{U}^{\left(\mathbb{A}_{L}\right)},
$$

where $\forall l \in[1, L], \mathbf{U}^{\left(\mathbb{A}_{l}\right)} \in \mathbb{C}^{\mathbb{A}_{l} \times \mathbb{A}_{l}}$ is an orthonormal matrix ${ }^{1}$. $\mathcal{K}_{\mathbb{A}_{1} / \ldots / \mathbb{A}_{L}} \in \mathbb{C}^{I_{1} \times \ldots \times I_{P}}$ is the core tensor which possesses the same properties as the HOSVD core tensor. $\times_{\mathbb{A}_{l}}$ is the multimode product ${ }^{2}$. There are multiple ways to decompose a tensor with this approach, depending of the choice of the partition $\mathbb{A}_{1}, \ldots, \mathbb{A}_{L}$. We proposed in the following section to design a LR MIMO STAP filter based on the AU-HOSVD.

\section{Extension of the orthogonal projector}

Let $\mathcal{H}, \mathcal{H}_{c}, \mathcal{H}_{0}$ be three $P$ th order tensors such that:

$$
\mathcal{H}=\mathcal{H}_{c}+\mathcal{H}_{0},
$$

where $\mathcal{H}_{c}$ is a $\left(r_{\mathbb{A}_{1}}, \ldots, r_{\mathbb{A}_{L}}\right)$ low rank tensor $\left(r_{\mathbb{A}_{l}}=\right.$ $\operatorname{rank}\left(\left[\mathcal{H}_{c}\right]_{\mathbb{A}_{l}}\right)$ ). Then $\mathcal{H}_{0}$ is approximated by:

$$
\mathcal{H}_{0} \approx \mathcal{H} \times_{\mathbb{A}_{1}} \mathbf{U}_{0}^{\left(\mathbb{A}_{1}\right)} \mathbf{U}_{0}^{\left(\mathbb{A}_{1}\right) H} \ldots \times_{\mathbb{A}_{L}} \mathbf{U}_{0}^{\left(\mathbb{A}_{L}\right)} \mathbf{U}_{0}^{\left(\mathbb{A}_{L}\right) H}
$$

where $\mathbf{U}_{0}^{\left(\mathbb{A}_{l}\right)}=\left[\begin{array}{llll}\mathbf{u}_{r_{\mathbb{A}_{l}}+1}^{\left(\mathbb{A}_{l}\right)} & \ldots & \mathbf{u}_{\mathbb{A}_{l}}^{\left(\mathbb{A}_{l}\right)}\end{array}\right]$. The same approximation can be applied to the HOSVD ${ }^{3}$.

\section{MIMO STAP}

We present in this section the model and the processing of the MIMO STAP data using firstly conventional approach and secondly multidimensional approach based on the AUHOSVD. We focus on LR filters for STAP in both vectorial and multidimensional approaches.

\section{A. Conventional MIMO STAP approach}

1) Signal model: We consider a MIMO radar with $M$ transmit antennas spaced by de and $N$ receive antennas spaced by $d r$. We assume that the radar system is in the coherent and colocated MIMO configuration, i.e. the transmit and the receive arrays are closely located to ensure that the received signal is coherent between the pairs of transmit and receive elements. We also consider $L$ pulses with a pulse repetition

\footnotetext{
${ }^{1} \mathbf{U}^{\left(\mathbb{A}_{l}\right)}$ is computed from the SVD of different kind of unfolding denoted []$_{\mathbb{A}_{l}}$. This allows to have a matrix of dimensions $\mathbb{A}_{l} \times \mathbb{A} \backslash \mathbb{A}_{l}$.

${ }^{2}$ This product is the extension of the $n$-mode product used for the HOSVD. It allows to multiply a tensor with a matrix along a combination of dimensions

${ }^{3}$ By analogy with HOSVD, even if the truncation for the AU-HOSVD is not optimal, we assume that it provides a correct approximation.
}

interval $T_{r}$. We assume that $M$ waveforms are ideally orthogonal and can then be perfectly disentangle at each receiver. In addition of the cell under test (CUT), we assume that $K$ observations are available. We assume that the CUT and the $K$ observation cells are independent and identically distributed. The received signal $\mathbf{x} \in \mathbb{C}^{M N L \times 1}$ for CUT and the received signal $\mathbf{x}_{k} \in \mathbb{C}^{M N L \times 1}$ for the $k$-th observation cell can be written as follows:

$$
\begin{aligned}
& \mathbf{x}=\alpha \mathbf{s}\left(f_{s}, f_{d}\right)+\mathbf{c}+\mathbf{n} \\
& \mathbf{x}_{k}=\mathbf{c}_{k}+\mathbf{n}_{k}
\end{aligned}, k \in[1, K],
$$

where $\alpha$ and $\mathbf{s}\left(f_{s}, f_{d}\right)$ are respectively the complex amplitude and the steering vector of the target, $\left(f_{s}, f_{d}\right)$ being the spatial and the Doppler frequencies of the target. $\mathbf{c}$ and $\mathbf{c}_{k}$ are the responses of the clutter with the following distribution $\mathcal{C N}\left(0, \mathbf{R}_{c}\right)$ with $\mathbf{R}_{c}$ the clutter covariance matrix. $\mathbf{n}$ and $\mathbf{n}_{k}$ are the responses of the thermal noise with the following distribution $\mathcal{C N}\left(0, \sigma^{2} \mathbf{I}_{M N L}\right)$ with $\sigma^{2}$ the power of the thermal noise.

As we consider ideally orthogonal waveforms and colocated MIMO, the steering vector $\mathbf{s}\left(f_{s}, f_{d}\right) \in \mathbb{C}^{M N L \times 1}$ is derived as follows [7]:

$$
\mathbf{s}\left(f_{s}, f_{d}\right)=\mathbf{a}\left(f_{s}\right) \otimes \mathbf{v}\left(f_{s}\right) \otimes \mathbf{b}\left(f_{d}\right)
$$

where

- $\quad \mathbf{a}\left(f_{s}\right)=\left[\begin{array}{llll}1 & \exp \left(j 2 \pi f_{s}\right) & \ldots & \exp \left(j 2 \pi(N-1) f_{s}\right)\end{array}\right]^{T}$ is the receive spatial steering vector,

- $\quad \mathbf{v}\left(f_{s}\right)=\left[1 \exp \left(j 2 \pi \gamma f_{s}\right) \ldots \exp \left(j 2 \pi \gamma(M-1) f_{s}\right)\right]^{T}$ is the transmit spatial steering vector with $\gamma=d e / d r$,

- $\mathbf{b}\left(f_{d}\right)=\left[\begin{array}{lll}1 & \exp \left(j 2 \pi f_{d} T_{r}\right) \quad \ldots & \exp (j 2 \pi(L-\end{array}\right.$ 1) $\left.\left.f_{d} T_{r}\right)\right]^{T}$ is the Doppler steering vector.

2) LR MIMO 2D STAP Filter: The goal of STAP is to efficiently reduce the clutter and noise responses for extracting the target response. The STAP methods initially developed for SIMO (Single Input Multiple Input), can be easily extended to the coherent MIMO with ideally orthogonal waveforms [6] by concatenating the responses of each transmitters for each receiver and each pulse.

It has been shown [5], [6] that the optimal STAP filter $\mathbf{w}_{\text {opt }}\left(f_{s}, f_{d}\right) \in \mathbb{C}^{M N L \times 1}$ is defined as:

$$
\mathbf{w}_{\text {opt }}\left(f_{s}, f_{d}\right)=\mathbf{R}^{-1} \mathbf{s}\left(f_{s}, f_{d}\right)
$$

where $\mathbf{R}=\mathbf{R}_{c}+\sigma^{2} \mathbf{I}_{M N L}$. As the covariance clutter-plusnoise matrix $\mathbf{R}$ is practically unknown, we use the estimate of this latter by using the $K$ additional observations $\hat{\mathbf{R}}=$ $\frac{1}{K} \sum_{k=1}^{K} \mathbf{x}_{k} \mathbf{x}_{k}^{\dagger}$. Usually the clutter subspace is low-rank and LR STAP methods have been developed to exploit this property [8], [7]. The LR STAP filter is based on the SVD of $\hat{\mathbf{R}}$. The rank $r$ of the clutter subspace can computed using the Generalized Brennan rule [7]. The SVD of $\hat{\mathbf{R}}$ is written as $\hat{\mathbf{R}}=\hat{\mathbf{U}} \hat{\boldsymbol{\Sigma}} \hat{\mathbf{V}}^{\dagger} . \hat{\mathbf{U}}_{0}=\left[\hat{\mathbf{u}}_{r+1} \ldots \hat{\mathbf{u}}_{N M L}\right]$ is a basis of the orthogonal of the clutter subspace, where $\hat{\mathbf{u}}_{i}$ are the $(N M L-r)$ last columns of $\hat{\mathbf{U}}$. The LR STAP filter $\hat{\mathbf{w}}_{L R}\left(f_{s}, f_{d}\right)$ and the output $y_{L R}\left(f_{s}, f_{d}\right)$ of this filter are written as follows:

$$
\begin{aligned}
& \hat{\mathbf{w}}_{L R}\left(f_{s}, f_{d}\right)=\hat{\mathbf{U}}_{0} \hat{\mathbf{U}}_{0}^{\dagger} \mathbf{s}\left(f_{s}, f_{d}\right) \\
& y_{L R}\left(f_{s}, f_{d}\right)=\hat{\mathbf{w}}_{L R}^{\dagger}\left(f_{s}, f_{d}\right) \mathbf{x}
\end{aligned}
$$


The LR methods allow to achieve good performances with a reduced number of secondary data (SINR loss ${ }^{4}$ of $3 \mathrm{~dB}$ for $K=2 r$ ) compared to fully adaptive STAP (SINR loss of $3 \mathrm{~dB}$ for $K=2 N M L)$.

\section{B. AU-Unfolding approach}

1) Signal Model: To derive and to apply the tensorial LR filter, we propose to rewrite the received signal model of Eq. (5) as follows:

$$
\begin{aligned}
& \boldsymbol{X}=\alpha \boldsymbol{S}\left(f_{s}, f_{d}\right)+\mathcal{C}+\mathcal{N} \\
& \boldsymbol{x}_{k}=\boldsymbol{C}_{k}+\mathcal{N}_{k}
\end{aligned} \quad k \in[1, K],
$$

where $\boldsymbol{X}, \boldsymbol{X}_{k} \in \mathbb{C}^{N \times M \times L}$ are the received signal tensors. $\boldsymbol{S}\left(f_{s}, f_{d}\right) \in \mathbb{C}^{N \times M \times L}$ is the steering tensor and is derived as follows:

$$
\boldsymbol{S}\left(f_{s}, f_{d}\right)=\operatorname{vec}^{-1}\left(\mathbf{s}\left(f_{s}, f_{d}\right)\right)
$$

where $\operatorname{vec}(\mathbf{Q})$ is the operator which transform a tensor $\mathbf{Q}$ into a vector $\mathbf{q}$. $v e c^{-1}$ is the inverse operator.

In the same way we derive the tensors of the clutter responses $\mathcal{C}, \mathcal{C}_{k} \in \mathbb{C}^{N \times M \times L}$ and the tensors of the thermal noise $\mathcal{N}, \mathcal{N}_{k} \in \mathbb{C}^{N \times M \times L}$. The clutter covariance tensor $\hat{\mathcal{R}} \in \mathbb{C}^{N \times M \times L \times N \times M \times L}$ is derived as follows:

$$
\hat{\mathcal{R}}=\operatorname{SqMat}^{-1}(\hat{\mathbf{R}})
$$

where $\operatorname{SqMat}(\mathcal{A})$ is the operator which transforms the square tensor $\mathcal{A} \in \mathbb{C}^{I_{1} \times I_{2} \times \ldots \times I_{P} \times I_{1} \times I_{2} \times \ldots \times I_{P}}$ into a square matrix $\mathbf{A} \in \mathbb{C}^{I_{1} I_{2} \ldots I_{P} \times I_{1} I_{2} \ldots I_{P}} . S q M a t^{-1}$ is the inverse operator.

2) Tensorial LR STAP filter: The tensorial LR STAP filter developed in [3], [4] is constructed in a similar way as the vectorial LR STAP filter. Based on the LR approximation of the AU-HOSVD in Eq. (4), the tensorial LR STAP filter $\hat{\mathcal{W}}_{l r\left(\mathbb{A}_{1}, \ldots, \mathbb{A}_{L}\right)}\left(f_{s}, f_{d}\right)$ and its output $y$ can be written as follows:

$$
\begin{gathered}
\hat{\mathcal{W}}_{l r\left(\mathbb{A}_{1}, \ldots, \mathbb{A}_{L}\right)}\left(f_{s}, f_{d}\right)=\boldsymbol{S}\left(f_{s}, f_{d}\right) \times_{\mathbb{A}_{1}} \hat{\mathbf{U}}_{0}^{\left(\mathbb{A}_{1}\right)} \hat{\mathbf{U}}_{0}^{\left(\mathbb{A}_{1}\right) \dagger} \times \\
\ldots \times_{\mathbb{A}_{L}} \hat{\mathbf{U}}_{0}^{\left(\mathbb{A}_{L}\right)} \hat{\mathbf{U}}_{0}^{\left(\mathbb{A}_{L}\right) \dagger} \\
y=\left|<\hat{\mathcal{W}}_{\mathbb{A}_{1}, \ldots, \mathbb{A}_{L}}, \boldsymbol{X}>\right|
\end{gathered}
$$

$\hat{U}^{\left(\mathbb{A}_{l}\right)}$ where $\hat{\mathbf{U}}_{0}^{\left(\mathbb{A}_{l}\right)}$ is derived from the AU-HOSVD of $\hat{\mathcal{R}}$, $\hat{\mathbf{U}}_{0}^{\left(\mathbb{A}_{l}\right)}=\left[\hat{\mathbf{u}}_{r_{\mathbb{A}_{l}}+1}^{\left(\mathbb{A}_{l}\right)} \ldots \hat{\mathbf{u}}_{\mathbb{A}_{l}}^{\left(\mathbb{A}_{l}\right)}\right]$ and $\left(r_{\mathbb{A}_{l}}=\operatorname{rank}\left([\hat{\mathcal{R}}]_{\mathbb{A}_{l}}\right)\right) .<,>$ is the scalar product operator.

3) Choice of the partition $\mathbb{A}$ : We only consider in this study the unfolding along the three dimensions of the data, i.e. along the $N$ receivers (dimension 1), the $M$ transmitters (dimension 2) and $L$ pulses (dimension 3). The different choices of partition are presented in table III-B3. The two first partitions can be seen as particular case of the AU-HOSVD. The first one $\mathbb{A}_{1}=\{1,2,3\}$ corresponds to the conventional vectorial STAP filter written in Eq. (8). The second one is the HOSVD. By combining two dimensions, we obtain the three last possible partitions. The ranks relative to the LR filters for each partition can be computed using the Generalized Brennan rule and other clutter rank computation rule. Due to a lack of space, the computation of the ranks is not studied here and will be proposed in a future paper.

\footnotetext{
${ }^{4}$ The SINR (Signal to Interference plus Noise Ratio) loss is the ratio between the SINR at the output of the STAP filter used and the SINR at the output of the optimal STAP filter.
}

\begin{tabular}{||c|l|c||}
\hline \hline Partition & Filters & methods \\
\hline $\mathbb{A}_{1}=\{1,2,3\}$ & $\hat{\mathcal{W}}_{l r(1,2,3)}=\hat{\mathbf{w}}_{L R}$ & Vector \\
\hline $\mathbb{A}_{1}=\{1\}, \mathbb{A}_{2}=\{2\}, \mathbb{A}_{3}=\{3\}$ & $\hat{\mathcal{W}}_{\operatorname{lr}(1 / 2 / 3)}$ & HOSVD \\
\hline $\mathbb{A}_{1}=\{1,2\}, \mathbb{A}_{2}=\{3\}$ & $\hat{\mathcal{W}}_{l r(1,2 / 3)}$ & AU-HOSVD \\
\hline $\mathbb{A}_{1}=\{1\}, \mathbb{A}_{2}=\{2,3\}$ & $\hat{\mathcal{W}}_{l r(1 / 2,3)}$ & AU-HOSVD \\
\hline $\mathbb{A}_{1}=\{1,3\}, \mathbb{A}_{2}=\{2\}$ & $\hat{\mathcal{W}}_{l r(1,3 / 2)}$ & AU-HOSVD \\
\hline \hline
\end{tabular}

TABLE I. DESCRIPTION OF THE LR FILTERS PROVIDED BY AU-HOS VD FOR MIMO STAP.

\section{Simulation RESUlts}

\section{A. Configuration}

We consider a colocated MIMO radar (and then coherent) with $M=4$ transmitters, $N=4$ receivers and $L=64$ pulses. The MIMO radar is in side-looking configuration. We simulate a ground clutter using Ward model [5] with $\beta=1$. We assume that the waveforms are ideally orthogonal and can then be perfectly disentangle at each receiver. At last, we consider two coherent MIMO geometrical configurations, MIMO sparse with $d r=\lambda_{0} / 2$ and $d e=N d r$ and MIMO filled with $d r=\lambda_{0} / 2$ and $d e=d r$ ( $\lambda_{0}$ is the wavelength of the transmit signals). The length of the virtual antenna array of the MIMO directly depends on the spacing between the transmit and the receive antennas. It has been shown [6] that the MIMO filled generates a virtual antenna array longer than the SIMO. The longest virtual antenna array is generated with the MIMO sparse. For comparison we also consider the SIMO (Single Input Multiple Input) configuration which is the conventional phased array with 4 receivers and 1 transmitters.

\section{B. SINR loss}

To evaluate the performances of the LR STAP filters based on the AU-HOSVD for MIMO, we perform simulations with 100 Monte Carlo runs. We present in Figure 1 and in Figure 2, the SINR losses in terms of the normalized Doppler frequencies for the SIMO using the LR STAP 2D filter and for the MIMO using the LR STAP 2D filter (LR filter) and the LR AU-HOSVD filters (AU-HOSVD) of each partition. We present results for the MIMO filled (MIMOf) in Figure 1 and for MIMO sparse (MIMOs) in Figure 2. The number of secondary data is equal to $2 r$ with $r$ being the rank of the clutter covariance matrix in the vectorial case $(r=67$ for SIMO, $r=70$ for MIMOf and $r=79$ for MIMOs).

We see in both figures that the SINR losses of the partitions $\{1,2 / 3\}$ and $\{1 / 2 / 3\}$ are lower than $-20 \mathrm{~dB}$ and then are not visible. For these two partitions, the spatial and the Doppler dimensions are not combined. Since the ranks for single dimension are not deficient, there is no projection into the orthogonal of the clutter subspace. Thus the suppression of the clutter is very bad. The vectorial LR filters for the SIMO and the MIMOf and MIMOs give the expected results with SINR losses around $-3 \mathrm{~dB}$; we also see that the clutter notch is narrower for the MIMO configurations compared to SIMO, especially for MIMOs which generates the longer virtual antenna. The two other partitions $\{1,3 / 2\}$ and $\{2,3 / 1\}$ combine one of the spatial dimension (transmitter or receiver) with the Doppler dimension. Compared to vectorial filters, the tensorial LR filters using these partitions give better results 
with almost the same number of secondary data with SINR loss around $-1 \mathrm{~dB}$. Nevertheless, the tensorial LR filters based on these partitions exploit only the physical transmit or receive antenna array (and not the virtual antenna array) as the two spatial dimensions are used separately. This means that the suppression of the clutter is only made on the receiver/Doppler dimensions or transmitter/Doppler dimensions. For the MIMOf in Figure 1, the LR filters based on the two partitions $\{1,3 / 2\}$ and $\{2,3 / 1\}$ have exactly the same performances because the transmit and the receive arrays are identical. Compared to the vectorial filter, the clutter notch of the tensorial filters are larger and equal to the SIMO. In MIMOs configuration in Figure 2 , the two partitions $\{1,3 / 2\}$ and $\{2,3 / 1\}$ do not have the same performances as the transmit and the receive arrays are different. For the partition $\{2,3 / 1\}$, the $\operatorname{LR}$ tensorial filter is derived using the combination of the transmit array and the Doppler dimension. As the transmit array is sparse, there are grating lobes which generate multiple narrow clutter notches in their positions. For the partition $\{1,3 / 2\}$, the LR tensorial filter is derived using the combination of the received array and the Doppler dimension; we obtain the same performance as for the MIMOf with a clutter notch as large as for the SIMO. Figure 3 presents the SINR losses versus the number

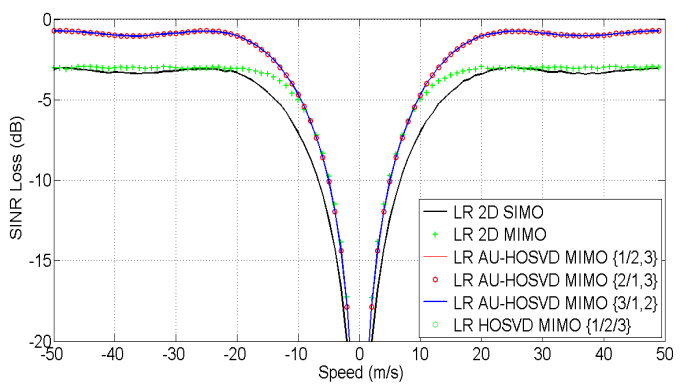

Fig. 1. SINR Losses versus Doppler frequency for each partition. MIMOf configuration.

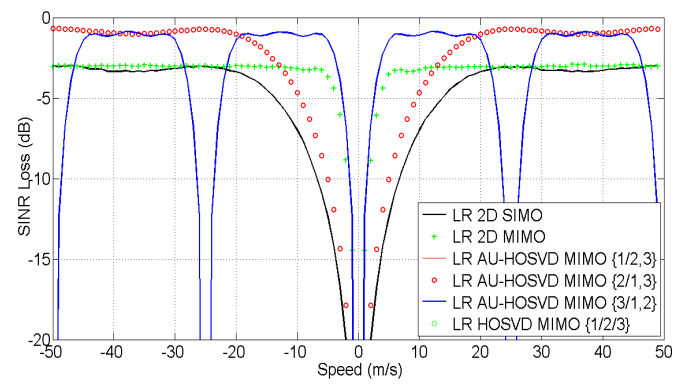

Fig. 2. SINR Losses versus Doppler frequency for each partition. MIMOs configuration.

of secondary data for a fast target located far from the clutter. We present the results of the LR 2D STAP filter for the SIMO and both MIMOf and MIMOs. We also present for both MIMO configurations, the results of LR STAP filter based on the AU-HOSVD of the partition $\{1,3 / 2\}$ (the other partitions do no give satisfying results for MIMO STAP). As we are far from the clutter notch, the maximum performance for all the cases and for a large number of secondary data, is the same and is approximately equal to $-1 \mathrm{~dB}$. We clearly see that the
SINR losses converge faster for both MIMO configurations using the LR filter based on the AU-HOSVD with less than 200 secondary data. Around the double of secondary data is needed to achieve a SINR loss of $-1 \mathrm{~dB}$ with the SIMO and the MIMOf and MIMOs using the LR 2D STAP.

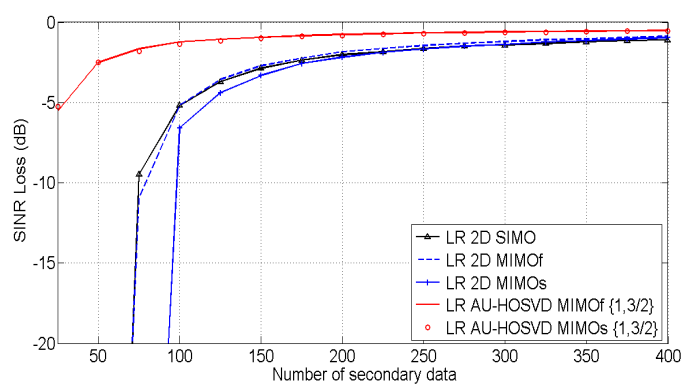

Fig. 3. SINR Losses versus the number of secondary data $(K)$. Fast target $\left(f_{s}=0, v=-40 m \cdot s^{-1}\right)$.

\section{CONCLUSION}

We have presented in this study a new LR filter for MIMO STAP based on multidimensional approach. The new decomposition obtained with the AU-HOSVD allows to process correlated data. We have chosen the most direct unfolding along the dimensions of the MIMO data. The STAP performances on simulated data are encouraging as we managed to reduce the number of secondary data needed to achieve good SINR losses. However, the chosen unfolding may not be the best one as we lose the generation of the virtual antenna for the suppression of the clutter. Further studies are needed, especially on the choice of the unfolding, in order to preserve the narrower clutter notch provided by the MIMO virtual antenna and the reduced number of secondary data provided by the AU-HOSVD approach.

\section{REFERENCES}

[1] T. Kolda and B. Bader, "Tensor decompositions and applications," SIAM Review, vol. 51, pp. 455 - 500, 2009.

[2] L. De Lathauwer, B. De Moor, and J. Vandewalle, "A multilinear singular value decomposition," SIAM J. Matrix Anal. Apl., vol. 24, no. 4, pp. 1253-1278, 2000

[3] M. Boizard, G. Ginolhac, F. Pascal, and P. Forster, "Low-rank filter and detector for multidimensional data based on an alternative unfolding HOSVD. application to polarimetric STAP," in IEEE Transactions on Signal Processing, 2013, Submitted.

[4] M. Boizard, G. Ginolhac, F. Pascal, and P. Forster, "A new tool for multidimensional low-rank STAP filter: Cross HOSVDs," in Proceedings of EUSIPCO, Bucharest, Romania, 2012.

[5] J. Ward, Space-time adaptive processing for airborne radar, Technical Report 1015, Massachusetts Institute of Technology, Lexington, MA, December 1994.

[6] J. Li and P. Stoica, MIMO Radar Signal Processing, Wiley, 1st edition, 2009.

[7] C.Y. Chen and P.P. Vaidyanathan, "MIMO radar space-time adaptive processing using prolate spheroidal wave functions," IEEE Transactions on Signal Processing, vol. 56, no. 2, pp. 623-635, February 2008.

[8] I.P. Kirsteins and D. Tufts, "Adaptive detection using a low rank approximation to a data matrix," IEEE Transactions on Aerospace and Electronic System, vol. 30, pp. 55 - 67, 1994. 